\title{
Based on PID and Neural network decoupling control system for special shape pipe automatic welding machine
}

\author{
MA Wen-bin, LIU Jian-hua \\ Mechanical Engineering, changshu institute of technology, Changshu china
}

Keywords: PID;neural network ;decoupling;special shape pipe welding

\begin{abstract}
In order to solve special shape pipe automatic welding problem,a new decoupling control system has been introduced,which is constructed on PID and RBF neural network.Input data is from non-contact displacement sensor and angle sensor,and output data has been used to control servo motor which always used to control the special shape pipe rotation and at the same time,the welding torch has also been controlled in order to regulate the distance from pipe to the font of torch.The simulation results show that the coupling system for special shaped pipe is effective.
\end{abstract}

\section{Introduction}

Special shaped pipe is widely used in industry.Through the purchase of special welding robot can ensure welding quality and improve work efficiency,but the cost is higher.Using the manual welding can not ensure the welding quality and the working efficiency.Therefore,by constructing a simple shape pipe automatic welding device to complete the welding shaped tube which can guarantee welding quality,and also have certain cost advantages.[1,2]

The key problem of special pipe welding equipment is how to solve the irregular shaped pipe welding process turn around decoupling control problem.Decoupling method are common:adaptive decoupling,decoupling robust and intelligent decoupling method. Yue Junhong was used improved predictive function decoupling controller design,simulation results show that the decoupling system can finish the decoupling task[1].Han Pu using muti-model predictive function to establish the main steam temperature control system[2].Xue Haoyang use of BP nerual network to establish a PID decoupling control of multi-variable system[3,4].

By optimizing the design of predictive functional control decoupling controller less influence the dynamic characteristics of the controlled object,easily obtained parameters, which has a certain practical value.[5,6,7]

I.The structure of the system

The unfinished welding shaped pipe section as shown in figure 1. The Special shape automatic welding mechanism contain 3-axis linkage welding implement,tow axis position, data detecting and processing part etc. During this process it is very important to keep a certain displacement between the welding gun and surface of the objects. By controlling 3-axis and 2-axis position changer the welding for special shape pipe would realize.

The data acquisition and processing part receive the information from non contact displacement sensor and rotary coder. The coder would detect degree of the object rotating during the welding. Then the data would be send to this part, and the welding machine will be controlled according to decoupling control algorithm which has been write within it before. This process has a key technology which is deal with the relationship between the welding gun position and the speed of the special shape pipe rounding. So the decoupling control of welding process is the key technology of it.[8] 


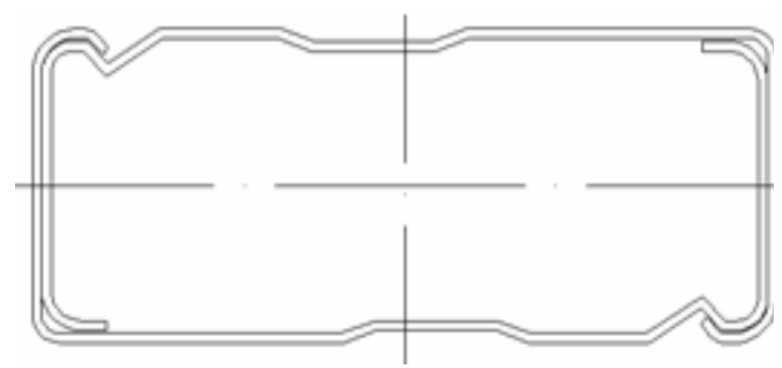

Figure 1 unfinished welding shape pipe

\section{2 .Decoupling control system model}

PID neural network from the structure is divided into the input layer, hidden layer and output layer, in each hidden layer contains adjustable parameters P, I and D three parameters, as shown in Figure 2[4].

$\mathrm{X} 11, \mathrm{X} 12, \mathrm{X} 21, \mathrm{X} 22$ for the decoupled system PID neural network input value, V1 and V2 is the output decoupling system values, Y1 and Y2 is the output value of the tooling system. Among them, $\mathrm{X} 11$ welding tooling turntable speed of servo motor; X12 is in accordance with the set welding tooling shape turntable servo motor running speed curve shaped tube; X21 automatic welding machine welding torch from the surface to be distance work piece setting value; X22 welding torch from the work piece surface to be distance from the actual value; V1 is the output decoupling system servo motor control, quantity; V2 for decoupling the output of the system, is the torch from the work piece surface distance control quantity; Y1 tooling dynamic model control output, for the servo motor speed; Y2 welding torch from the surface of the work piece size;[9]

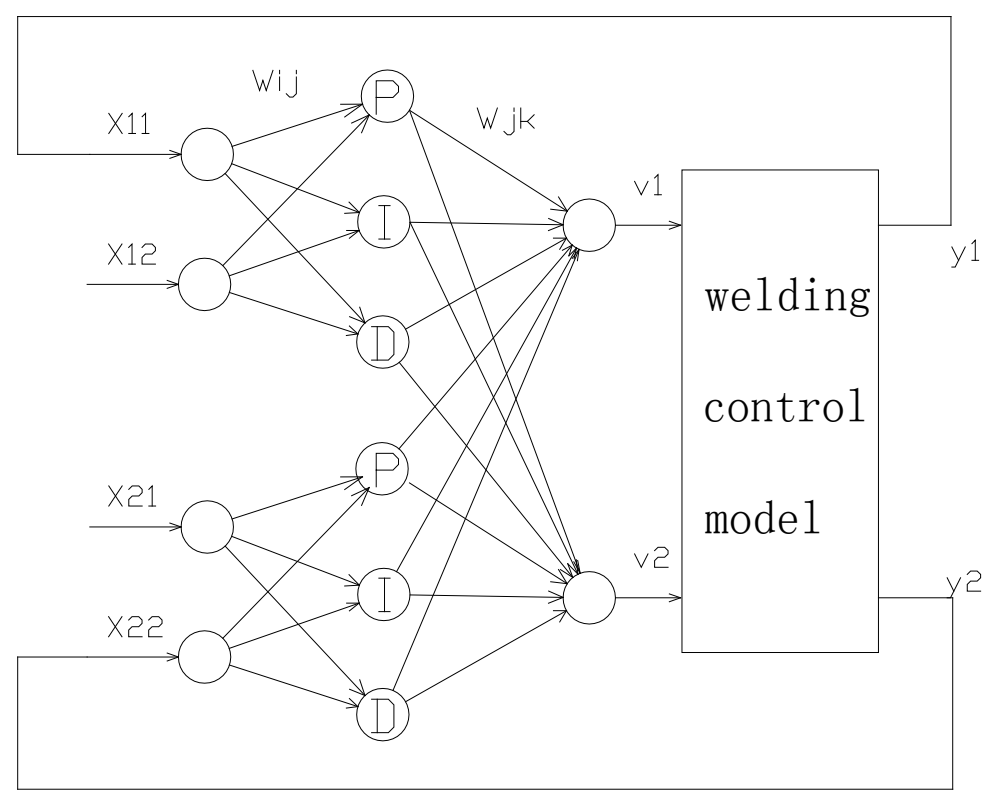

Figure 2 PID decoupling system structure

\subsection{PID decoupling model}

In a sampling time of $\mathrm{K}$, input and output functions of neurons in the input layer of the PID neural network can be expressed as[5]:

$$
x_{s i}(k)=u_{s i}(k)
$$

Among them, as the input layer neuron input; input and output layer neuron; The output layer:

$$
x_{h}^{\prime \prime}(k)=\sum_{s=1}^{2} \sum_{j=1}^{3} \omega_{s j h}^{\prime} x_{s j}^{\prime}(k)
$$

Among them, as the connection weights of hidden layer and output layer is the output layer neuron number. 


$$
J=\sum_{p=2}^{2} E_{p}=\frac{1}{m} \sum_{p=1}^{2} \sum_{k=1}^{m}\left[r_{p}(k)-y_{p}(k)\right]^{2}=\frac{1}{m} \sum_{p=1}^{2} \sum_{k=1}^{m} e_{p}^{2}(k)
$$

Train process own gradient descent algorithm. The hidden layer to the output layer weights training adjustment formulas can be expressed by the following formula group:

$$
\omega_{s j h}^{\prime}\left(n_{0}+1\right)=\omega_{s j h}^{\prime}\left(n_{0}\right)-\eta_{s j h}^{\prime} \frac{\partial J}{\partial \omega_{d j h}^{\prime}}
$$

The input layer to the hidden layer calculation formula can be expressed as[9,10]:

$$
\begin{gathered}
\omega_{s i j}\left(n_{0}+1\right)=\omega_{s i j}\left(n_{0}\right)-\eta_{s i j} \frac{\partial J}{\partial \omega_{s i j}} \\
\frac{\partial \mathrm{J}}{\partial \omega_{s i j}}=-\frac{1}{m} \sum_{p=1}^{n} \sum_{h=1}^{n} \sum_{k=1}^{m}\left[\delta_{h p}^{\prime}(k) \omega_{s j h}^{\prime} \operatorname{sgn} \frac{x_{s j}^{\prime}(k)-x_{s j}^{\prime}(k-1)}{u_{s j}^{\prime}(k)-u_{s j}^{\prime}(k-1)} x_{s i}(k)\right]
\end{gathered}
$$

\subsection{Welding tooling dynamic model}

The establishment of process dynamic model: output by PID neural network decoupling system: the calculated servo motor speed control quantity and the torch from the work piece surface distance control quantity for training the RBF neural network input that system; servo motor output speed and welding torch from the size of the work piece surface dynamic model is established for the RBF neural network output. Among them, the output of the RBF network input from the PID neural network, the input of PID neural network and RBF network from the set value of the output.

\section{Algorithm simulation}

Based on the MATLAB platform, construct the simulation model of PID and RBF neural

\begin{tabular}{|c|c|c|c|c|c|}
\hline $\begin{array}{l}\text { ang } 1 \\
\mathrm{e} /\end{array}$ & $\begin{array}{l}\text { Displace } \\
\text { ment/mm }\end{array}$ & ang $1 \mathrm{e}$ & $\begin{array}{l}\text { Displace } \\
\text { ment/mm }\end{array}$ & angle & $\begin{array}{c}\text { Displacem } \\
\text { ent/mm }\end{array}$ \\
\hline 5 & 23.1 & 10 & 23.3 & 15 & 23.5 \\
\hline 20 & 24.06 & 25 & 24.9 & 30 & 25.5 \\
\hline 35 & 25.9 & 40 & 27.8 & 45 & 29.6 \\
\hline 50 & 34. 1 & 55 & 37.2 & 60 & 46 \\
\hline 65 & 59 & 70 & 62.9 & 75 & 62.3 \\
\hline 80 & 61.2 & 85 & 60.7 & 90 & 60.2 \\
\hline
\end{tabular}
network.In the tracking target by shaped as drawing a given size tube contour shape, set sampling points is 500, the network control variable to 2 step 0.01. Part of the turntable angle and torch position data such as shown in Table 1, decoupling control curve as shown in Figure 3

Table 1 Part of the turntable angle and the welding torch position data 

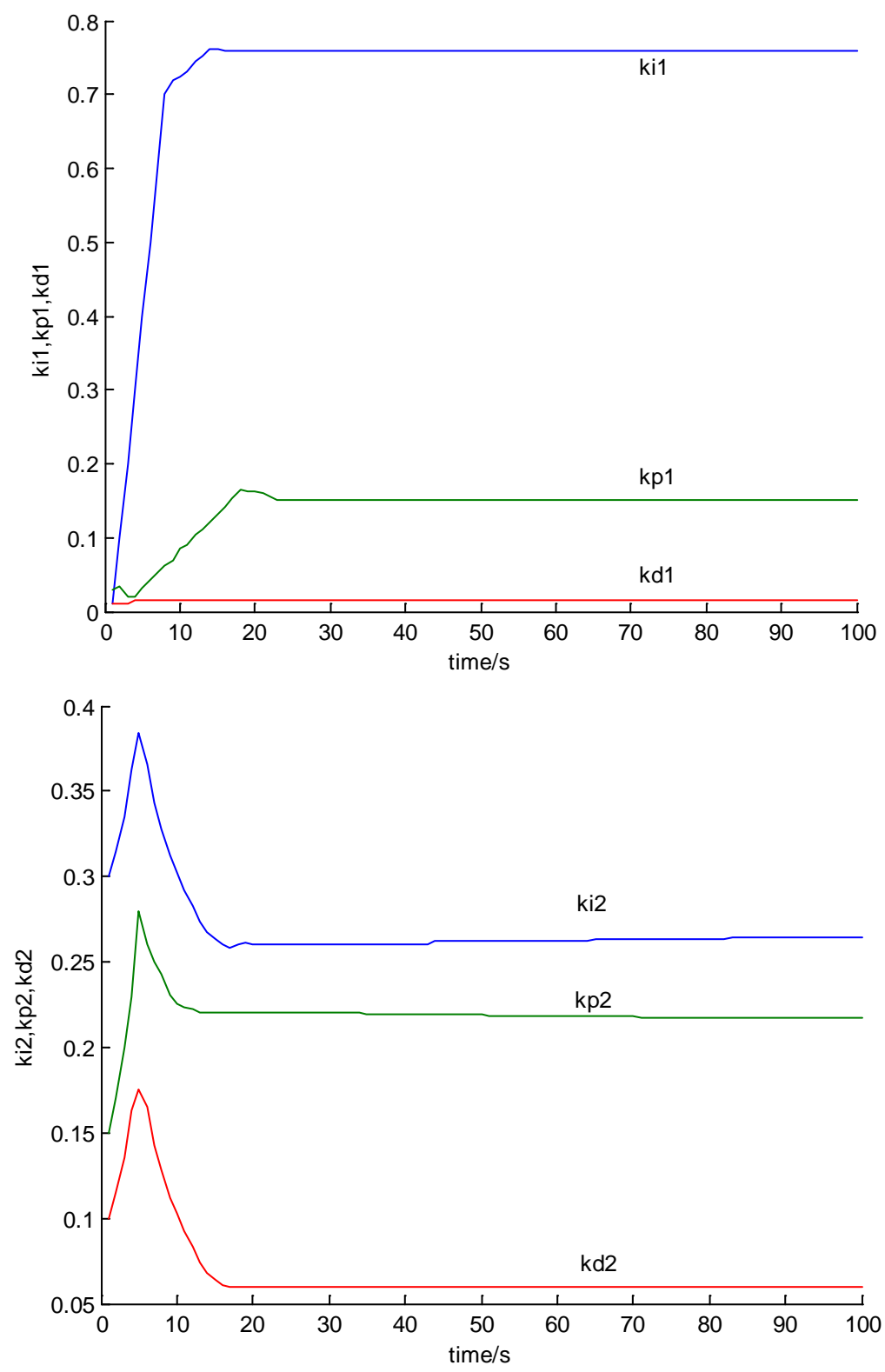

Figure.3 PID1 PID2parameter response curve

Simulation of PID neural network setting value as shown in Figure 3 and the output value, the RBF controller output values as shown in figure 4.

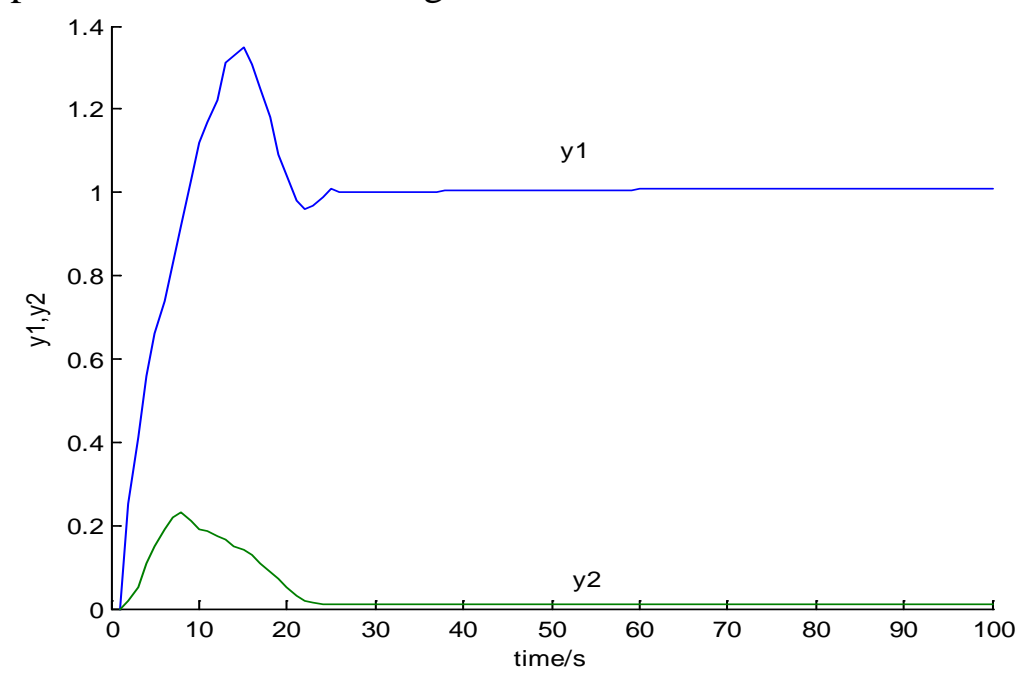

Figure 4 The controller output response curve 


\section{4 conclusion}

The simulation results show that the RBF neural network, decoupling multivariable PID controller can complete the control of multivariable systems based on PID, compared with the traditional decoupling control, the adaptive ability and anti disturbance of the obvious advantages.

\section{References}

[1]Shu Huai-lin.Inverted Pendulum Control System Based on PID Neural Network.Machine Tool and Hydraulics.Vol.36(3),2008.3:141-146

[2]Fu Long-hai,Li Meng.Variable-air-volume Air-Conditioning system based on PID-ANN decoupling Control Technology.Journal of southwest jiaotong university.Vol.40(1),2005(2):13-17;

[3]Zaheer-uddin M,Zheng G R.A dynamic model of a mulit-zone VAV system for control analsisi.ASHRAE Trans 1994,100(1):219-229

[4]Akhyar,S.,Omatu S. Self-tuning PID control by neural-networks.IEEE Transactions on Power Systems,Vol 3,25-29 Oct 1993:2749-2752

[5]Zhang Jie,Zou Ji-gang,Li Wen-xiu.PID neural Network for Decoupling Control of Multi-input and Multi-output system.Journal of Harbin engineering University.Vol.21(5)2000(10):6-9

[6] Leung F H F,Lam H K,Ling S H,et al.Tuning of the structure and parameters of a neural network using an improved genetic algorithm. IEEE Transactions on Neural Networks . 2003

[7] Lu YW,Sundararajan N,Saratchandran P.A sequential learning scheme for function approximation using minimal radial basis function (RBF) neural networks. Neural Computation . 1997

[8] Sheng Chen,Andreas Wolfgang,,Chris J Harris,,Lajos Hanzo.Symmetric RBF Classifier for Nonlinear Detection in Multiple-Antenna-Aided Systems. IEEE Transactions on Neural Networks . 2008

[9] Chen S,Wang X X,Brown D J.Sparse incremental regression modeling using correlation criterion with boosting search. IEEE Signal Processing Letters . 2005

[10] Guang-Bin Huang,P. Saratchandran,Narasimhan Sundararajan.A Generalized Growing and Pruning RBF (GGAP-RBF) Neural Network for Function Approximation. IEEE Transactions on Neural Networks . 2005 